\title{
Friction and Dissipation in Epitaxial Graphene Films
}

\author{
T. Filleter, ${ }^{1}$ J. L. McChesney, ${ }^{2}$ A. Bostwick, ${ }^{2}$ E. Rotenberg, ${ }^{2}$ K. V. Emtsev, ${ }^{3}$ Th. Seyller, ${ }^{3}$ K. Horn, ${ }^{4}$ and R. Bennewitz ${ }^{1,5, *}$ \\ ${ }^{1}$ Department of Physics, McGill University, Montreal, Quebec H3A 2T8, Canada \\ ${ }^{2}$ Advanced Light Source, Lawrence Berkeley National Laboratory, Berkeley, California 94720, USA \\ ${ }^{3}$ Lehrstuhl für Technische Physik, Universität Erlangen-Nürnberg, 91058 Erlangen, Germany \\ ${ }^{4}$ Department of Molecular Physics, Fritz-Haber-Institut der Max-Planck-Gesellschaft, 14195 Berlin, Germany \\ ${ }^{5}$ INM -Leibniz Institute for New Materials, 66123 Saarbrücken, Germany
}

(Received 27 November 2008; published 27 February 2009)

\begin{abstract}
We have studied friction and dissipation in single and bilayer graphene films grown epitaxially on SiC. The friction on $\mathrm{SiC}$ is greatly reduced by a single layer of graphene and reduced by another factor of 2 on bilayer graphene. The friction contrast between single and bilayer graphene arises from a dramatic difference in electron-phonon coupling, which we discovered by means of angle-resolved photoemission spectroscopy. Bilayer graphene as a lubricant outperforms even graphite due to reduced adhesion.
\end{abstract}

Understanding the fundamental origin of friction is one of the great challenges in tribology. At the core of the problem is an identification of the mechanisms that convert kinetic energy of sliding contacts into heat [1]. The difficulty arises from the significant complexity found at the sliding interface, making an identification of the fundamental mechanisms involved in friction elusive. Recently this challenge has been met by approaches which reduce complexity to shed light onto two fundamental mechanisms of energy dissipation in friction: i.e., electronic contributions and phononic contributions [2,3]. These studies have demonstrated how friction changes upon an exclusive variation of the electronic and vibrational properties of a surface, respectively.

In this Letter, we study fundamental processes in friction and dissipation in a model system of both technological relevance and physical fascination. By reducing the thickness of the solid lubricant graphite to the most extreme limits, single and double atomic layers of graphene, we demonstrate that a significant difference in friction between single and bilayer graphene films exists. We correlate this difference to a dramatic reduction in electronphonon coupling in the bilayer as revealed by angleresolved photoemission spectroscopy.

Graphene, the single planar sheet of hexagonally coordinated carbon, exhibits a wealth of unique electronic transport properties [4-7]. Graphene is, however, not only interesting in terms of electrical transport, but it is also the building block of the most common macroscopic solid lubricant graphite. The pseudo-two-dimensional $s p^{2}$ bonding in graphite yields one of the most effective solid lubricants: the ease of shearing or delaminating the individual 2D graphene planes, weakly bonded in the third dimension by van der Waals forces, has been traditionally used to explain the excellent lubricating properties of graphite [8]. However, the friction properties of graphene planes merit investigation, since single and bilayer graphene films provide a means of investigating both the microscopic origins of friction and the intrinsic tribological properties of graphite by reducing it to its fundamental limit.

The epitaxial growth of graphene supported by a semiconducting substrate may provide a technologically relevant system to take advantage of the exciting electrical properties of graphene [9]. This could also lead to an equally interesting mechanical analog: graphene films as a solid lubricant on $\mathrm{SiC}$ substrates, an emerging material in the design of microelectromechanical systems [10].

Single (1LG) and bilayer (2LG) graphene films were prepared ex situ on $\mathrm{SiC}(0001)$ using thermal decomposition under atmospheric pressure to produce large graphene terraces [11]. The local layer thickness of the films has been identified by means of Kelvin probe force microscopy, taking advantage of reproducible differences in the local contact potential of the carbon-rich interface, 1LG and $2 \mathrm{LG}$ [12]. Frictional properties of the films were measured using the friction force microscopy (FFM) mode of a homebuilt UHV-AFM system. With our apparatus, we can accomplish three important steps with the same probe tip: (i) identify the surface structure and layer thickness using Kelvin probe force microscopy in a noncontact AFM (nc-AFM) mode; (ii) then switch to FFM mode to conduct local friction measurements; (iii) finally reimage the sample in nc-AFM mode to ensure we are working in a zero-wear regime. In order to run measurements in both modes with the same probing tip we use cantilevers which have both a high enough normal resonance frequency $(f \sim 80 \mathrm{kHz})$ to allow for stable nc-AFM imaging as well as low normal and lateral stiffness $\left(k_{N} \sim\right.$ $2 \mathrm{~N} / \mathrm{m}, k_{L} \sim 400 \mathrm{~N} / \mathrm{m}$ ) to allow for sensitive FFM measurement. For the data presented here we have used cantilevers coated in a polycrystalline diamond film (Nanosensors) [13].

We determine the total dissipated energy upon sliding the tip across the sample by measuring the lateral force as a function of position as the tip is dragged back and forth 
across a boundary between adjacent $1 \mathrm{LG}$ and $2 \mathrm{LG}$ terraces [Fig. 1(a)]. In Fig. 1(a) the tip slides across both layer thicknesses on either side of the boundary step within a single scan line. The total dissipated energy during the round trip is given by the enclosed area of the hysteresis loop, and the shaded areas reflect the relative amount of energy dissipated in the $1 \mathrm{LG}$ and $2 \mathrm{LG}$ regions. Clearly the energy dissipation rate, and therefore friction, is lower on $2 \mathrm{LG}$ regions as compared to $1 \mathrm{LG}$; it differs by about a factor of 2, an effect that is found at all loads. Figure 1(b) shows a plot of the average lateral force vs normal loading for $1 \mathrm{LG}$ and $2 \mathrm{LG}$ films. The average lateral force on each layer is determined from a portion of each forward and backward trace, as indicated in Fig. 1(a), away from the transition region to avoid artifacts in the frictional force at the step edge. These results have been reproduced with several different tips. We find that the slope of the load dependence can vary for different tips but the ratio of the lateral force measured on $1 \mathrm{LG}$ and $2 \mathrm{LG}$ is always consistent. The variation in slope can easily be explained by different tip sizes, again stressing the necessity of simultaneous measurements. The constant ratio precludes an assignment of the difference in friction to adhesion contrast between $1 \mathrm{LG}$ and $2 \mathrm{LG}$.

A number of extrinsic effects that can cause friction contrasts across heterogeneous surfaces can also be ruled out based on the well-defined nature of our sample and atomic-scale friction experiments. Any effect of a changing tip structure, geometrically or chemically, can be neglected due to the simultaneous recording of $1 \mathrm{LG}$ and $2 \mathrm{LG}$ data. Atomic stick-slip friction measurements on 1LG and 2LG films confirm a consistent factor of 2 difference in average friction [see shaded regions in Figs. 1(e) and 1(f)]. The contact stiffness extracted from stick-slip friction loops shows no change between layers which could otherwise indicate compliance differences and affect the average lateral force. Furthermore, atomic resolution confirms that the orientation of the lattice is the same for both layer thicknesses [Figs. 1(c) and 1(d)], excluding the dramatic effect of varying orientational commensurability between tip and sample [14]. This is expected for a topmost graphene film which grows continuously over substrate steps and covers the underlying transition between $1 \mathrm{LG}$ and $2 \mathrm{LG}$ [15]. Potentially, the higher friction on 1LG could be caused by higher interfacial forces between tip apex and $1 \mathrm{LG}$ as compared to $2 \mathrm{LG}$. The atomic stick-slip results described above exclude such contrast as expected for films of identical atomic structure. These findings suggest that the nature of the contrast in frictional properties arises not from variations in the tip-surface contact but from an intrinsic energy dissipation mechanism difference between single and bilayer graphene.

For an interpretation of the intrinsic friction difference between single and bilayer graphene established by the above data, let us first consider whether the differences in electronic structure can affect friction. For the as-grown 1LG and 2LG films, the doping level is equal [16], thus ruling out any direct connection between doping level and friction. Studies of doped Si and GaAs have demonstrated that friction can be tuned by electric fields between tip and
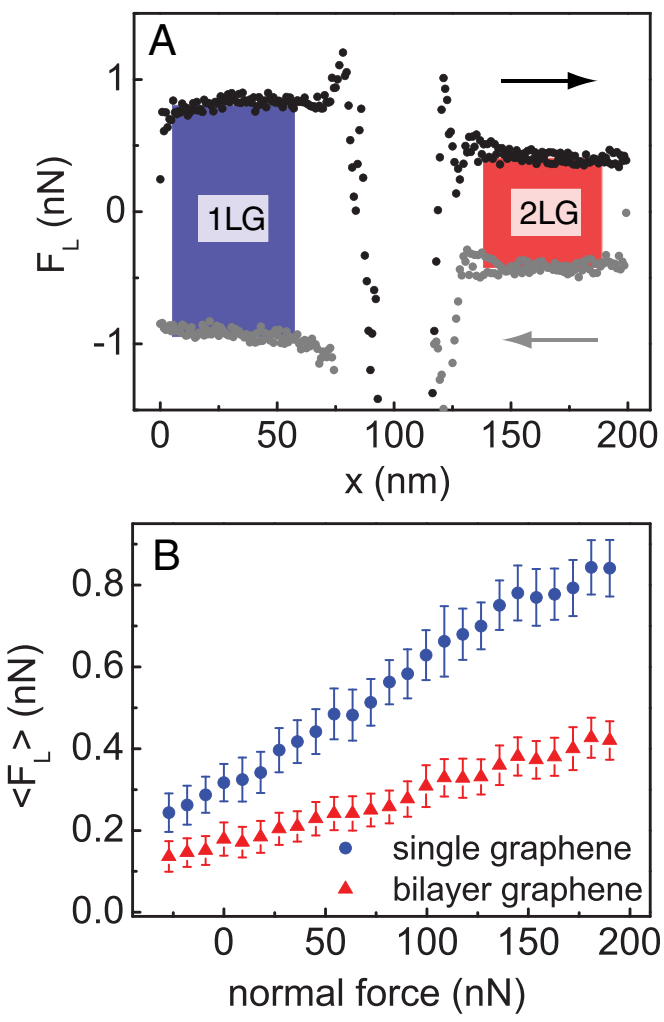
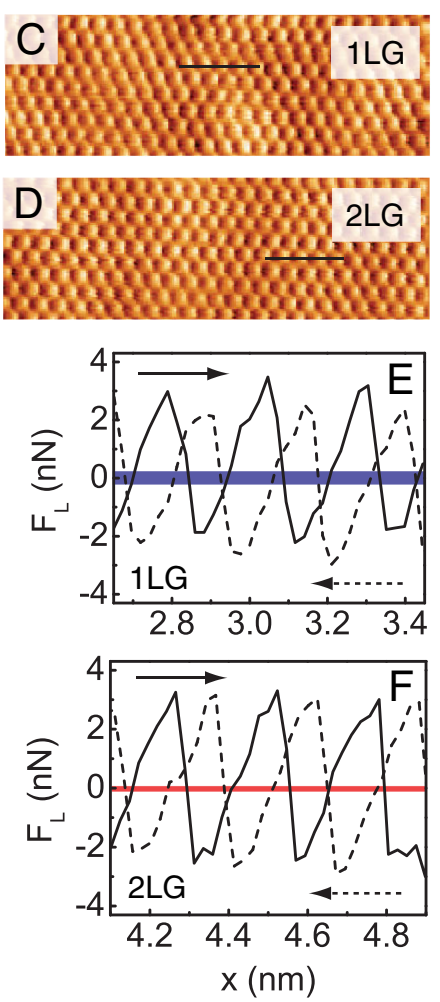

086102-2
FIG. 1 (color online). (a) Friction loop (averaged over 50 lines) recorded on a boundary region of the sample with adjacent areas of single (1LG) and bilayer (2LG) films at a normal load of $172 \mathrm{nN}$. (b) Average lateral force as a function of normal load simultaneously recorded for 1LG and 2LG. (c),(d) Atomic stick-slip friction force maps recorded on adjacent 1LG and 2LG films (image size, $6 \times$ $2 \mathrm{~nm}^{2}$; normal load, $181 \mathrm{nN}$; scan rate, $30 \mathrm{~nm} / \mathrm{s}$ ). (e),(f) Corresponding lateral force line profiles (positions indicated in friction force maps). The shaded regions indicate the average lateral force. 
sample $[2,17]$. In these studies the dominant contribution to excess friction has been attributed to the tip-induced hopping of trapped charges in the oxide layer, where a threshold bias is required to initiate the population of these trap states. In the current work the friction contrast between graphene layers is observed at zero bias, thereby excluding this effect. Furthermore, we have repeated the friction experiments with biases compensating the contact potential of the interface layer, the single and bilayer films, respectively, and found no change in the friction contrast.

In order to identify intrinsic differences in $1 \mathrm{LG}$ and $2 \mathrm{LG}$ films, we have performed angle-resolved photoemission spectroscopy (ARPES), which directly probes many-body interactions in solids [18]. ARPES measures the band structure as a single-particle spectral function $A(\mathbf{k}, \omega)$, where $\mathbf{k}$ is the electron momentum and $\omega$ is the electron binding energy. Many-body interactions manifest themselves in $A(\mathbf{k}, \omega)$ by changes of the $\omega$-dependent linewidth of the bands (measuring the quasiparticle scattering rates) accompanied by a renormalization of the bands' energies.

We have previously shown that the carrier lifetime in 1LG is determined by a combination of electron-electron $(e-e)$, electron-phonon ( $e-\mathrm{ph})$, and electron-plasmon $(e-\mathrm{pl})$ interactions [19]. As the only mechanisms for inelastic scattering, changes in any of these interactions can in principle explain the observed difference in dissipation and hence in friction [1]. Here we give evidence for a dramatic difference in the $e$-ph coupling between 1LG and 2LG by determining the energy renormalization of the bands.

Figures 2(a) and 2(d) show a comparison of the ARPES spectral function $A(\mathbf{k}, \omega)$ determined from $1 \mathrm{LG}$ and $2 \mathrm{LG}$. The data were acquired near the $\pi^{*}$ band crossing of the Fermi energy $E_{F}$. At $\sim 200 \mathrm{meV}$ binding energy, the $1 \mathrm{LG}$ band shows a kink attributable to $e$-ph coupling $[19,20]$. The kink is a manifestation of the enhanced mass of the carriers near $E_{F}$, and its strength can be characterized by the maximum difference between the renormalized band energy $\omega_{r}(\mathbf{k})$ and the unrenormalized, linear bare band $\omega_{b}(\mathbf{k})$, which occurs around $\omega_{r}(\mathbf{k})=165 \mathrm{meV}$.

For 2LG, the presence or absence of an $e$-ph kink is hard to assess for the as-grown films, since the $\pi$ bands are gapped by the electric field at the interface [16,21], and hence the $\pi^{*}$ band's width is only slightly greater than the phonon energy scale. Therefore, we have added small amounts of $\mathrm{K}$ atoms in order to increase the occupied $\pi^{*}$ bandwidth as previously described [16,19,21]. Figures 2(b) and 2(e) show representative results for doped 1LG and $2 \mathrm{LG}$, respectively. What is remarkable is that an $e$-ph kink, while present, is almost undiscernible for the slightly doped 2LG film while it is quite clear for a $1 \mathrm{LG}$ film at similar doping. Upon further $\mathrm{K}$ deposition, the kink becomes clearly visible for $2 \mathrm{LG}$, but it remains about half the strength as the kink in the 1LG film [Figs. 2(c) and 2(f)].

The changes in $e$-ph coupling strength with doping are summarized in Figs. 2(g) and 2(h), where we have plotted the difference $\omega_{r}(\mathbf{k})-\omega_{b}(\mathbf{k})$ vs $\omega$, which is an estimate of the real part of the electron self-energy [20]. As doping is reduced from the maximum level considered, the kink strength in both 2LG and 1LG decrease, since the phase space for $e$-ph scattering decreases as the Fermi surface shrinks. But the phonon kink weakens more dramatically for $2 \mathrm{LG}$ than for $1 \mathrm{LG}$, becoming undetectable for the asgrown film used in the friction measurements.

This lead us to the most likely scenario to explain the increased friction in $1 \mathrm{LG}$ as compared to $2 \mathrm{LG}$. Phonons excited by the mechanical energy of the sliding tip in the 1LG are much more likely to scatter with electrons, introducing a more efficient means of dissipating energy. Since the phonon distribution of $1 \mathrm{LG}$ and $2 \mathrm{LG}$ are similar (as evidenced by the similar shape of the phonon kinks), the difference in scattering rate appears not to be due to an increased number of flexural modes in 1LG but to fundamental differences in their $e$-ph coupling constants that remain to be explained.

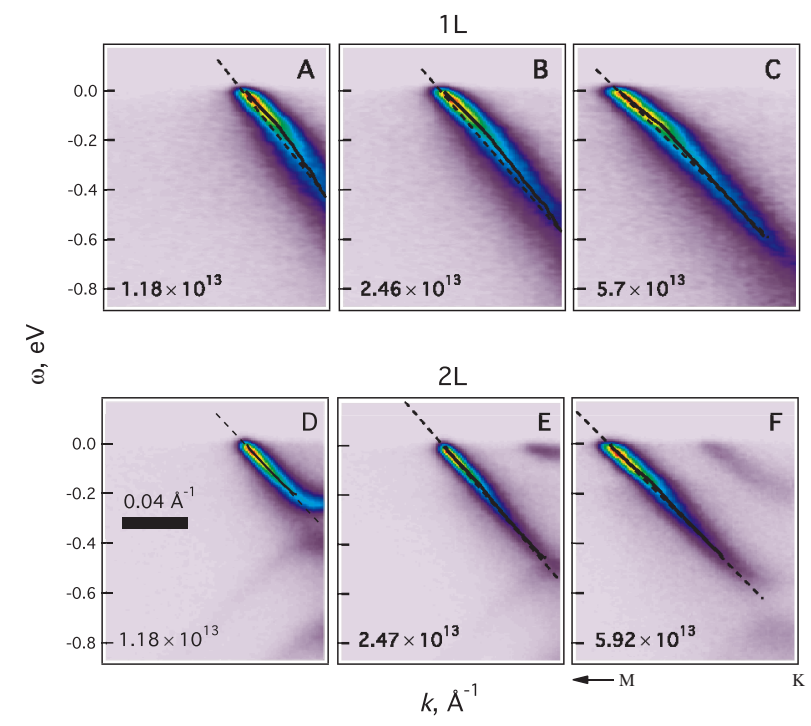

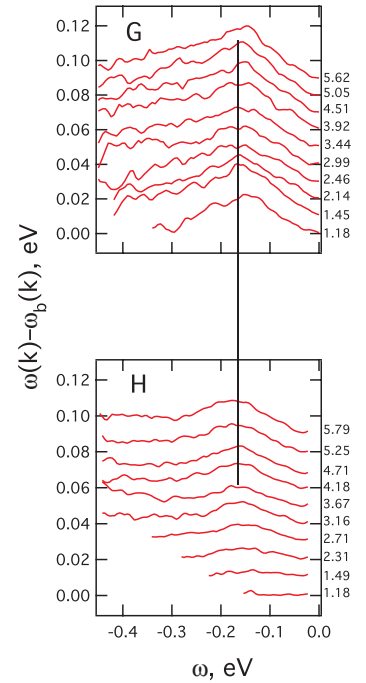

086102-3
FIG. 2 (color online). Kinks in the band structure of single (1LG) and bilayer (2LG) graphene. (a)-(c) Spectral function $A(\mathbf{k}, \omega)$ for $2 \mathrm{LG}$ three dopings (in $e^{-} / \mathrm{cm}^{2}$ ) as indicated. The dashed lines are linear bare bands $\omega_{b}(\mathbf{k})$, the solid heavy lines are the fitted band positions $\omega_{r}(\mathbf{k})$. (d)-(f) Similar, for 1LG. (g),(h) Corresponding kink magnitude $\omega_{r}(\mathbf{k})-\omega_{b}(\mathbf{k})$ for 1LG then 2LG as a function of doping values indicated (in units of $10^{13} e^{-} / \mathrm{cm}^{2}$ ). 


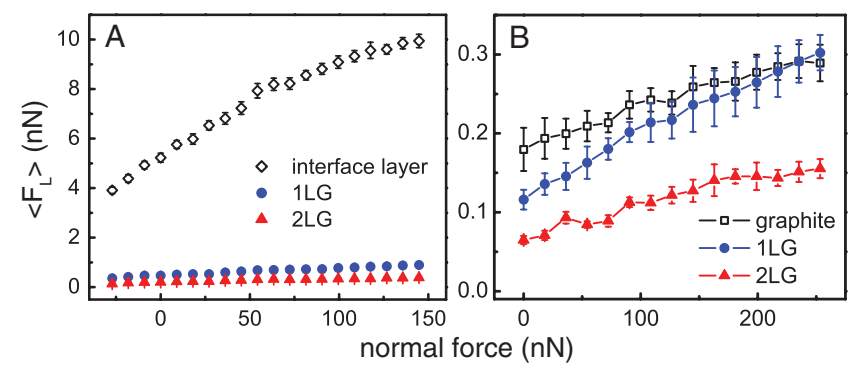

FIG. 3 (color online). (a) Plot of the average lateral force as a function of normal force simultaneously recorded on the carbonrich interface layer, single (1LG), and bilayer (2LG) graphene. (b) Normal vs average lateral force measured using the same AFM tip averaged over repeated measurements on graphite, $1 \mathrm{LG}$, and 2LG.

We propose the following physical picture for the difference in friction between $1 \mathrm{LG}$ and $2 \mathrm{LG}$ films: during the observed stick-slip-type sliding motion, the lattice is locally distorted and released by the slipping probe tip transferring kinetic energy into lattice vibrations. In the case of the $1 \mathrm{LG}$ film, the associated lattice motion is damped by the creation of electronic excitations through $e$-ph coupling, much more so than in the case of $2 \mathrm{LG}$ films where the $e$-ph coupling is vanishingly small. This additional mechanism increases the efficiency of energy dissipation during slip events which are responsible for frictional energy losses. Microscopically, we do not expect that the lifetime of excited lattice vibrations extends from one slip to the next. However, it is well know that the atomic slip events are quite complex: Thermal fluctuations let the tip jump back and forth between atomic positions [22], double slips over two lattice constants may occur [23], and the extended contact may undergo consecutive partial slips [24]. For 2LG, undamped lattice vibrations excited during such complex slip events reduce the average lateral force required for the tip to slide over the surface, similar to the way in which thermal vibrations reduce friction [22]. For 1LG, however, lattice vibrations are more efficiently damped, thereby removing this vibrational reduction of lateral force and dissipating more energy in the sample in the form of electron excitation. Within this picture $e$-ph coupling found in $1 \mathrm{LG}$ but not $2 \mathrm{LG}$ can account for the factor of 2 difference between frictional dissipation in $1 \mathrm{LG}$ and 2LG films at all loads whereas the increase of friction with load is a result of a stronger lateral surface potential and increasing contact area.

Finally, we would like to bring the friction results for the graphene films into a materials perspective. Figure 3(a) compares the friction on both graphene films and the carbon-rich interface layer [15]. Friction is lower by an order of magnitude as compared to the interface layer. This suggests that graphene films could be used to further reduce friction on $\mathrm{SiC}$ surfaces which are already technologically used as a low-friction antiwear coating. When compared to bulk graphite using the same tip we find that graphene films exhibit quite similar frictional properties. Figure 3(b) shows a plot of lateral force vs normal load for graphite, $1 \mathrm{LG}$ and $2 \mathrm{LG}$. The slope of the load dependence of friction for the bilayer graphene and graphite are the same. However, graphite has higher friction due to a loadindependent offset which indicates a stronger adhesion. The increased adhesion may be attributed to a larger contact area of the more compliant bulk graphite. Single layer graphene has a steeper load dependence than graphite, but for low loads still shows lower friction than graphite due to low adhesion.

This work was supported by the Canada Foundation of Innovation and NSERC. Research at the ALS was supported by the Director, Office of Science, Office of Basic Energy Sciences, of the U.S. Department of Energy. J.L.M. and K.H. were supported by the Max Planck Society. Research in Erlangen was supported by the DFG and within the Cluster of Excellence "Engineering of Advanced Materials."

*roland.bennewitz@inm-gmbh.de

[1] B. Persson, Sliding Friction, NanoScience and Technology (Springer, Berlin, 2000), 2nd ed.

[2] J. Park et al., Science 313, 186 (2006).

[3] R. Cannara et al., Science 318, 780 (2007).

[4] K. S. Novoselov et al., Science 306, 666 (2004).

[5] K. S. Novoselov et al., Proc. Natl. Acad. Sci. U.S.A. 102, 10451 (2005).

[6] Y. Zhang et al., Nature (London) 438, 201 (2005).

[7] A. Geim et al., Nature Mater. 6, 183 (2007).

[8] W. Bollmann et al., Nature (London) 186, 29 (1960).

[9] C. Berger et al., Science 312, 1191 (2006).

[10] C. Stoldt et al., Sens. Actuators. A Phys. 97-98, 410 (2002).

[11] K. V. Emtsev et al., Nature Mater. (to be published).

[12] T. Filleter et al., Appl. Phys. Lett. 93, 133117 (2008).

[13] Results have also been reproduced using oxidized single crystal silicon cantilevers with sharper tips.

[14] M. Dienwiebel et al., Phys. Rev. Lett. 92, 126101 (2004).

[15] P. Lauffer et al., Phys. Rev. B 77, 155426 (2008).

[16] T. Ohta et al., Phys. Rev. Lett. 98, 206802 (2007).

[17] Y. Qi et al., Phys. Rev. B 77, 184105 (2008).

[18] A. Damascelli et al., Rev. Mod. Phys. 75, 473 (2003).

[19] A. Bostwick et al., Nature Phys. 3, 36 (2007).

[20] J. L. McChesney et al., arXiv:0809.4046.

[21] T. Ohta et al., Science 313, 951 (2006).

[22] S. Krylov et al., New J. Phys. 9, 398 (2007).

[23] S. Medyanik et al., Phys. Rev. Lett. 97, 136106 (2006).

[24] S. Maier et al., Phys. Rev. B 72, 245418 (2005). 